\title{
Hypercoagulability and coronary artery disease
}

\author{
N T J O'CONNOR, S CEDERHOLM-WILLIAMS, S COPPER, L COTTER \\ From the Departments of Haematology and Cardiology, Fohn Radcliffe Hospital, Oxford
}

SUMMARY Variables of haemostatis were studied in 21 men, aged less than 50 years, with confirmed coronary artery disease but without severe hyperlipidaemia and in 21 healthy controls. Fibrinogen concentrations were significantly raised in the patients, $10(48 \%)$ of whom also showed defective fibrinolysis in response to a standard stress test. These findings suggest that hypercoagulability may be important in the aetiology of some cases of early onset coronary artery disease.

A hypercoagulable state may be an important factor in the pathogenesis of coronary artery disease, ${ }^{1}$ although its relative significance has not been fully defined. We studied variables of haemostasis and fibrinolytic potential in a group of young men with coronary artery disease but without severe hyperlipidaemia. By studying young subjects and excluding those with hyperlipidaemia requiring treatment with drugs we hoped to highlight abnormalities in our study group.

Subjects, methods, and results

We studied 21 men, aged less than 50 years who had angiographic evidence of coronary artery disease. Seventy one such men underwent coronary angiography between 1 January 1979 and 31 December 1983 to assess whether patients with angina pectoris were suitable for surgical intervention, but those who had undergone surgery in the previous six months, lived more than $\mathbf{3 0}$ miles away, or were considered by their cardiologist to be psychologically unsuitable for study were excluded, as were three patients with hyperlipidaemia requiring treatment with drugs. No patient had diabetes mellitus. Twenty one healthy nonsmoking men aged 25 to 48 were included.as control subjects.

All 21 patients were well, and none had been in hospital during the previous 12 months. At angiography all patients were shown to have severe coronary artery disease with complete occlusion of at least one coronary artery in seven patients and almost complete occlusions in 12. Myocardial infarction had been

Requests for reprints to Dr N T J O'Connor, Department of Haematology, John Radcliffe Hospital, Headington, Oxford OX3 9DU.

Accepted for publication 23 July 1984 documented in four patients, and 13 had undergone coronary artery surgery. Thirteen continued to receive antianginal treatment (seven with beta blockade alone), but only four suffered from exertional angina. No patient was taking coumarin anticoagulants, and only two patients were smokers at the time of study, both smoking fewer than 10 cigarettes a day. Four patients had borderline hypertriglyceridaemia. The Table compares the patients and controls.

The study was carried out between 1 June and 28 November 1983, with each subject being examined at least twice at a minimum interval of two weeks. Subjects were seen under standard conditions to minimise physiological variations in fibrinolytic activity. ${ }^{2}$ All subjects were seen between 0830 and 1030, after an overnight fast, with blood being drawn after 30 minutes' rest in the supine position. Samples were assayed for prothrombin ratio, kaolin cephalin clotting time, fibrinogen concentrations, full blood count, and fasting blood lipid concentrations using standard techniques. Plasminogen activator activities were then assayed with euglobulin lysis on fibrin plates before and after a venous occlusion stress test to determine the subject's fibrinolytic potential. ${ }^{3}$

Full blood count, prothrombin time ratio, and kaolin cephalin clotting times were within the normal range for all subjects. Plasminogen activator response to venous occlusion was generally consistent between the two visits, with the higher plasminogen activator activity after venous occlusion being taken to reflect the subject's fibrinolytic potential. Fig. 1 shows the range of responses, with a plasminogen activator activity after venous occlusion of less than $0.5 \mathrm{Com}$ mittee Thrombolytic Agents Units (CTA)/ml reflecting poor fibrinolytic potential. ${ }^{3}$ Ten of the 21 patients showed poor fibrinolysis compared with only two of the 21 controls $(p<0.05$ by Fisher's exact test). Fib- 
Table Data on 21 young men with corbnary artery disease and on 21 healthy controls

\begin{tabular}{|c|c|c|c|c|c|c|}
\hline & $\begin{array}{l}\text { Mean (range) } \\
\text { age (yr) }\end{array}$ & No of smokers & $\begin{array}{l}\text { No weighing } \\
>120 \% \text { ideal }\end{array}$ & $\begin{array}{l}\text { No with mild } \\
\text { hypertension }\end{array}$ & $\begin{array}{l}\text { Mean (SD) fasting } \\
\text { triglyceride (mmol/l) }\end{array}$ & $\begin{array}{l}\text { Mean (SD) fasting } \\
\text { cholesterol (mmol/l) }\end{array}$ \\
\hline $\begin{array}{l}\text { Patients }(n=21) \\
\text { Controls }(n=21)\end{array}$ & $\begin{array}{l}47.8(37-49) \\
33.7(25-48)\end{array}$ & 2 & $\begin{array}{l}4 \\
1\end{array}$ & $\begin{array}{l}2 \\
1\end{array}$ & $\begin{array}{l}1.60(0.66) \dagger \\
0.73(0.33)\end{array}$ & $\begin{array}{l}6.18(0.95) \\
4.93(0.90)\end{array}$ \\
\hline
\end{tabular}

$\star$ Diastolic blood pressure $95-100 \mathrm{~mm} \mathrm{Hg}$.

tOnly one patient had fasting triglyceride $>2.3 \mathrm{mmol} / \mathrm{l}$ (at $3.5 \mathrm{mmol} / \mathrm{l}$ ).

Conversion: SI to traditional units-Triglyceride: $1 \mathrm{mmol} / \approx \approx 88.6 \mathrm{mg} / 100 \mathrm{ml}$. Cholesterol: $1 \mathrm{mmol} / \mathrm{l} \approx 38.7 \mathrm{mg} / 100 \mathrm{ml}$.

rinogen concentrations were considerably increased in the patients with coronary artery disease compared with the controls $(\mathrm{p}<0.001$ by unpaired two tailed $t$ test), as Fig. 2 shows.

\section{Discussion}

We found early onset coronary artery disease to be

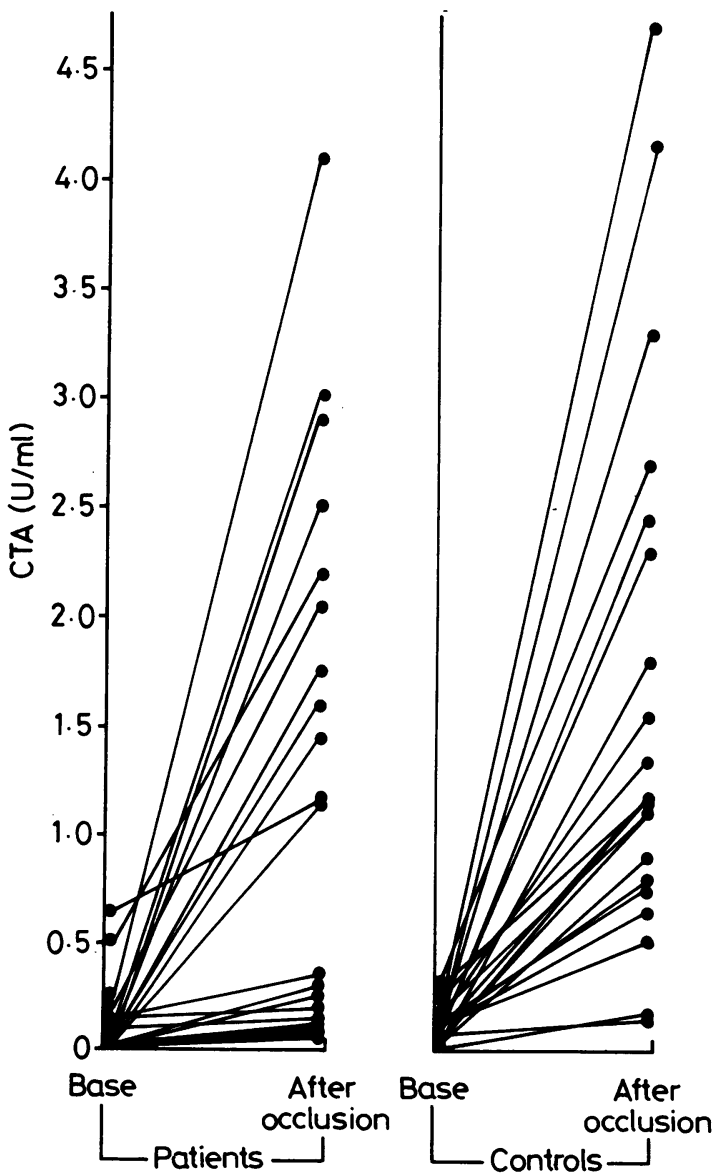

Fig. 1 Response of plasminogen activator (Committee Thrombolytic Agents Units/ml) to venous occlusion in 21 young men with confirmed coronary artery disease and in 21 controls. associated with both defective fibrinolysis and high fibrinogen concentrations in our study population. The association of a hypercoagulable state with coronary artery disease is not explained by shared risk factors as patients and controls were matched for body weight, blood pressure, and smoking habits. Differences in fasting triglyceride concentrations were slight, with nearly all patients having values lying within the quoted "normal range." The disparity in age between the two groups (patients' mean age 47.8 years compared with controls' mean age 33.7 years)

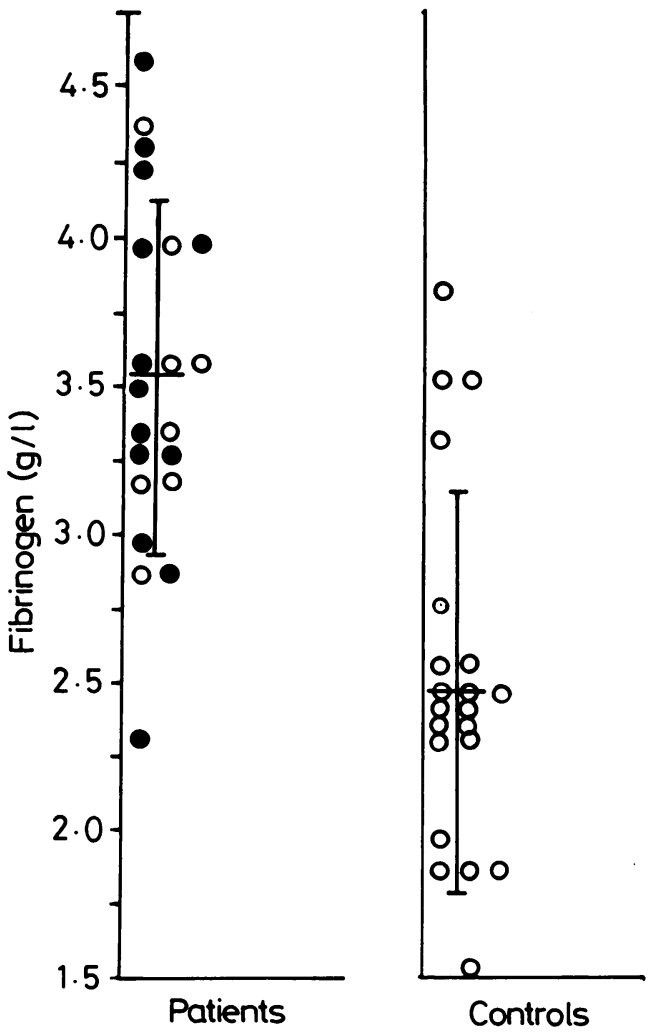

Fig. 2 Fibrinogen concentrations in 21 men aged below 50 with confumed coronary artery disease and in 21 controls. (O) Patients receiving regular antianginal treatment. (O) Subjects receiving no drug treatment. 
does not explain the observed differences in haemostatic variables because fibrinogen concentrations rise only slowly with age, ${ }^{4}$ and fibrinolytic potential improves as subjects become older. ${ }^{3}$

Although fibrinolytic activity is difficult to assess, the response in plasminogen activator activity to standard venous occlusion is sensitive, repeatable, and of confirmed clinical relevance in the study of patients with venous thromboembolic disease. ${ }^{23}$ Fibrinolytic potential was reduced in $10(48 \%)$ of our patients compared with in only two (9.5\%) of the controls and the expected incidence of reduced fibrinolytic potential in the general population is around $5 \% .^{3}$ These differences are not explained by drug treatment because beta blockers have no effect on the fibrinolytic system. ${ }^{2}$ The evidence for poor fibrinolytic potential being a risk factor for coronary artery disease is not clear, but our work supports an earlier suggestion that coronary artery disease in the young may often be associated with defective fibrinolysis. ${ }^{56}$ It is of note that smoking and obesity-two positive risk factors for coronary artery disease-depress fibrinolytic potential, but exercise and alcohol promote it and are cardioprotective. ${ }^{2}$ The severity of the coronary artery disease was not more pronounced in those patients with defective fibrinolysis.

The Northwick Park Heart Study showed that increased concentrations of fibrinogen and coagulation factors VII and VIII are associated with an increased risk of death from cardiovascular disease but failed to show any association with defective fibrinolysis. ${ }^{1}$ Our work confirms their finding that fibrinogen concentrations are appreciably higher in patients with coronary artery disease than in controls but also suggests that defective fibrinolysis may be a significant risk factor for coronary artery disease. This supports the proposition that many patients with early onset coronary artery disease have an associated "hypercoagulable" state.

We thank Dr B Gribbin and Professor P Sleight for allowing us to study their patients; the Oxford Area Research Committee for support; and Dr T E A Peto for criticism of the report.

\section{References}

1 Meade TW, Chakrabarti R, Haines AP, et al. Haemostatic function and cardiovascular death: early results of a prospective study. Lancet 1980; i: 1050-4.

2 Marsh N. Normal fibrinolysis: common causes of variation. In: Fibrinolysis. Chichester: John Wiley and Sons, 1981: 92-124.

3 Nilsson IM. Coagulation, fibrinolysis and venous thrombosis. Triangle 1977; 16: $19-27$.

4 Meade TW, Chakrabarti R, Haines AP, North WRS, Stirling Y. Characteristics affecting fibrinolytic activity and plasma fibrinogen concentrations. $\mathrm{Br} M e d \mathcal{F} 1979$; i: 153-6.

5 Chakrabarti R, Hocking ED, Fearnley GR, Mann RD, Attwell TN, Jackson D. Fibrinolytic activity and coronary-artery disease. Lancet 1968; i: 987-90.

6 Walker ID, Davidson JF, Hutton I, Lawrie TDV. Disordered "fibrinolytic potential" in coronary heart disease. Thromb Res 1977; 10: 509-20. 